\title{
FOURIER SERIES AND EIGENFUNCTION EXPANSIONS ASSOCIATED WITH A NON-SELF-ADJOINT DIFFERENTIAL EQUATION*
}

\author{
BY \\ LUNA I. MISHOE \\ Delaware State College
}

1. Introduction. In a paper by B. Friedman and L. Mishoe [1] ${ }^{* *}$ it is shown that if $u_{1}(x)$ and $u_{2}(x)$ are fundamental solutions of the non-self-adjoint equation

$$
u^{\prime \prime}+q(x) u+\lambda\left[p(x) u-u^{\prime}\right]=0
$$

and $v_{1}(x)$ and $v_{2}(x)$ corresponding solutions of the adjoint equation

$$
v^{\prime \prime}+q(x) v+\lambda\left[p(x) v+v^{\prime}\right]=0,
$$

then for $f(x)$ of bounded variation on $(0,1)$ and $0<x<1$ the series

$$
\sum_{-\infty}^{\infty} u_{n}(x) \int_{0}^{1} f(\xi) \frac{p(\xi) v_{n}(\xi)+v_{n}^{\prime}(\xi)}{C^{\prime}\left(\lambda_{n}\right)} d \xi \equiv \sum_{-\infty}^{\infty} a_{n} u_{n}(x)
$$

converges to

$$
\frac{1}{2}[f(x+)+f(x-)]-\frac{1}{2}[f(0+)+f(1-) \exp [-P(0,1)]] \exp [P(0, x)],
$$

where $u_{n}(x)$ and $v_{n}$ are the eigenfunctions of (1) and (2) associated with the eigenvalues $\lambda=\lambda_{n} . C(\lambda)=w(0, \lambda)$ where $w(x, \lambda)$ is the Wronskian associated with the fundamental solutions $u_{1}$ and $u_{2}$. The $\lambda_{n}$ are the zeros of $C(\lambda)$ and

$$
P(\xi, x) \equiv \int_{\xi}^{x} p(t) d t .
$$

In the expansion (3) the function $f(x)$ was required to be of bounded variation on $(0,1)$.

In this paper we generalize the result of [1] by allowing $f(x)$ to belong to the Class $L^{2}(0,1)$ and then proving that $\sum_{-\infty}^{\infty} a_{n} u_{n}(x)$ is convergent or uniformly convergent with the Fourier series

$$
\sum_{-\infty}^{\infty} \exp (2 n \pi i x) \int_{0}^{1} f(\xi) \exp (-2 n \pi i \xi) d \xi \equiv \sum_{-\infty}^{\infty} b_{n} \exp (2 n \pi i x) ;
$$

thus connecting expansions associated with non-self-adjoint differential equations with the study of Fourier series.

We express our result more precisely in the following theorem. $\dagger$

2. Theorem. If $u_{n}(x)$ are eingenfunctions associated with the system

$$
u^{\prime \prime}+q u+\lambda\left(p u-u^{\prime}\right)=0, \quad u(0)=u(1)=0,
$$

and $p^{\prime \prime}(x)$ exists and $q(x)$ is continuous on $(0,1)$ then the series $\sum_{-\infty}^{\infty} a_{n} u_{n}(x)$ is convergent

*Received August 18, 1960; revised manuscript received December 4, 1961.

**In [1] $H(x, \lambda)$ and $J(x, \lambda)$ are denoted as $\gamma(x, \lambda)$ and $\theta(x, \lambda)$ respectively.

†Similar equiconvergence theorems for Fourier series and self-adjoint eigenfunction expansions are well known e.g. see Ref. [2], Vol. 2, pp. 771-772 and Ref. [3], pp. 276-278. 
or uniformly convergent with the Fourier series $\sum_{-\infty}^{\infty} b_{n} \exp (2 n \pi i x)$ for $0<x<1$ and $f(x)$ of Class $L^{2}(0,1)$. Furthermore if $\sum_{-\infty}^{\infty} b_{n} \exp (2 n \pi i x)=t(x)$ then

$$
\sum_{-\infty}^{\infty} a_{n} u_{n}(x)=t(x)-t_{0} \exp [P(0, x)],
$$

where

$t_{0}=\sum_{-\infty}^{\infty} \int_{0}^{1}[f(\xi) \exp [-2 x P(0,1)+P(0, x)]] \exp (-2 n \pi i \xi) d \xi$ and $P(\xi, x) \equiv \int_{\xi}^{x} p(t) d t$.

3. Proof of the theorem. If we let

$$
\psi_{1}=p v_{1}+v_{1}^{\prime}, \quad \psi_{2}=p v_{2}+v_{2}^{\prime}, \quad H(x, \lambda)^{*}=\frac{u_{2}(x)}{C(\lambda)} \int_{0}^{x} f(\xi) \psi_{1}(\xi) d \xi
$$

and

$$
J(x, \lambda)=\frac{u_{1}(x)}{C(\lambda)} \int_{x}^{1} f(\xi) \psi_{2}(\xi) d \xi,
$$

it is shown in [1] that

$$
\begin{aligned}
\sum_{-\infty}^{\infty} u_{1}\left(x, \lambda_{n}\right) \int_{0}^{1} f(\xi)\left[\frac{p(\xi) v_{2}\left(\xi, \lambda_{n}\right)+v_{2}^{\prime}\left(\xi, \lambda_{n}\right)}{C^{\prime}\left(\lambda_{n}\right)}\right] d \xi & \\
= & \sum_{-\infty}^{\infty} u_{2}\left(x, \lambda_{n}\right) \int_{0}^{1} f(\xi)\left[\frac{p \xi) v_{1}\left(\xi, \lambda_{n}\right)+v_{1}^{\prime}\left(\xi, \lambda_{n}\right)}{C^{\prime}\left(\lambda_{n}\right)}\right] d \xi \\
& =\frac{1}{2 \pi i} \oint H(x, \lambda) d \lambda+\frac{1}{2 \omega i} \oint J(x, \lambda) d \lambda=\sum_{-\infty}^{\infty} a_{n} u_{n}(x),
\end{aligned}
$$

where the contour is a circle with radius tending to infinity [1].

Asymptotic evaluations for $H$ and $J$ have been determined in [1], where it is shown that

$$
\begin{gathered}
H(x, \lambda)=\left[\frac{\exp [\lambda(x-1)-P(1, x)]-\exp [P(1, x)]+0(1 / \lambda)}{\exp [-P(0,1)]-\exp [-\lambda+P(0,1)]+0(1 / \lambda)}\right] \\
\cdot \int_{0}^{x} f(\xi)\left[\exp [-\lambda \xi+P(0, \xi)]+O\left(\frac{\exp [-\lambda \xi]}{\lambda}\right)+O\left(\frac{1}{\lambda^{2}}\right)\right] d \xi \\
\text { for } \operatorname{Re} \lambda>0 \\
H(x, \lambda)=\left[\frac{\exp [P(1, x)]-\exp [\lambda(1-x)+P(1, x)]+0(1 / \lambda)}{\exp [\lambda-P(0,1)]-\exp [P(0,1)]+0(1 / \lambda)}\right] \\
\cdot \int_{0}^{x} f(\xi)\left[\exp [\lambda(x-\xi)+P(0, \xi)]+O\left(\frac{\exp [\lambda(x-\xi)]}{\lambda}\right)\right] d \xi \\
J(x, \lambda)=\left[\frac{\exp [-P(0, x)]-\exp [-\lambda x+P(0, x)]+0(1 / \lambda)}{\exp [-P(0,1)]-\exp [-\lambda+P(0,1)]+0(1 / \lambda)} \lambda<0\right. \\
\cdot \int_{x}^{1} f(\xi)\left[\exp [\lambda(x-\xi)+P(1, \xi)]+O\left(\frac{\exp [\lambda(x-\xi)]}{\lambda}\right)\right] d \xi
\end{gathered}
$$


$$
J(x, \lambda)=\left[\frac{\exp [\lambda x-P(0, x)]-\exp [P(0, x)]+0(1 / \lambda)}{\exp [\lambda-P(0,1)]-\exp [P(0,1)]+0(1 / \lambda)}\right]
$$$$
\text { for } \operatorname{Re} \lambda>0 \text {, }
$$$$
\cdot \int_{x}^{1} f(\xi)\left[\exp [\lambda(1-\xi)+P(1, \xi)]+O\left(\frac{\exp [\lambda(1-\xi)]}{\lambda}\right)\right] d \xi
$$$$
\text { for } \operatorname{Re} \lambda<0 \text {. }
$$

Since $f(x)$ is $L^{2}(0,1)$ we may apply the Schwarz inequality and obtain

Similarly

$$
\left|\int_{0}^{x} f(\xi) O\left(\frac{1}{\lambda^{2}}\right) d \xi\right| \leq\left(\int_{0}^{x} f^{2}(\xi) d \xi \int_{0}^{x}\left[0\left(\frac{1}{\lambda^{2}}\right)\right]^{2} d \xi\right)^{1 / 2}=0\left(\frac{1}{\lambda^{2}}\right) .
$$

$$
\int_{0}^{x} f(\xi) O\left(\frac{\exp -\lambda \xi}{\lambda}\right) d \xi=O\left(\frac{\exp -\lambda x}{\lambda}\right) .
$$

It is shown in [1] that if $C_{1}$ is the semicircle for which $\operatorname{Re} \lambda>0$ and whose radius tends to infinity then

Also

$$
\int_{C_{2}} O\left(\frac{\exp -\lambda \xi}{\lambda}\right) d \lambda=0 \text { and } \int_{\Gamma .} 0\left(\frac{1}{\lambda^{2}}\right) d \lambda=0
$$

$\lim _{|\lambda| \rightarrow \infty}\left[\frac{\exp [\lambda(x-1)-P(1, x)]-\exp [P(1, x)]+0(1 / \lambda)}{\exp [-P(0,1)]-\exp [-\lambda+P(0,1)]+0(1 / \lambda)}\right]$

and we conclude that

$$
=-\exp [P(0, x)] \text { for } \operatorname{Re} \lambda>0
$$

$$
\begin{aligned}
\frac{1}{2 \pi i} \int_{C_{1}} H(x, \lambda) d \lambda=- & \frac{\exp [P(0, x)]}{2 \pi i} \\
& \cdot \int_{C_{1}} \int_{0}^{x} f(\xi) \exp [-\lambda \xi+P(0, \xi)] d \xi d \lambda \text { for } f(x) L^{2}(0,1)
\end{aligned}
$$

and similarly as in [1]

$$
\begin{aligned}
& \frac{1}{2 \pi i} \int_{C_{2}} H(x, \lambda) d \lambda=-\frac{\exp [P(0, x)]}{2 \pi i} \int_{C_{2}} \int_{0}^{x} f(\xi) \exp [\lambda(x-\xi)+P(0, \xi)] d \xi d \lambda \\
& \frac{1}{2 \pi i} \int_{C_{2}} J(x, \lambda) d \lambda=\frac{\exp [P(x, 1)]}{2 \pi i} \int_{C_{1}} \int_{x}^{1} f(\xi) \exp [\lambda(x-\xi)+P(1, \xi)] d \xi d \lambda \\
& \frac{1}{2 \pi i} \int_{C_{2}} J(x, \lambda) d \lambda=\frac{\exp [-P(x, 1)]}{2 \pi i} \int_{C_{2}} \int_{x}^{1} f(\xi) \exp [\lambda(1-\xi)+P(1, \xi)] d \xi d \lambda
\end{aligned}
$$

where $C_{2}$ is the semi-circle for which $\operatorname{Re} \lambda<0$ and whose radius tends to infinity.

Consider now the new functions $\mu_{1}(x, \lambda), \mu_{2}(x, \lambda)$ and $K(\lambda)$ derived from $u_{1}(x, \lambda)$, $u_{2}(x, \lambda)$ and $C(\lambda)$ by dropping all order terms [1]. We have

$$
\begin{aligned}
\mu_{1} & =\frac{\exp [\lambda x-P(0, x)]-\exp [P(0, x)]}{\lambda} \\
\mu_{2} & =\frac{\exp [\lambda(x-1)-P(1, x)]-\exp [P(1, x)]}{\lambda}, \\
K(\lambda) & =\frac{\exp [-P(0,1)]-\exp [-\lambda+P(0,1)]}{\lambda} .
\end{aligned}
$$


It is easy to see that $K(\lambda)$ has zeros at $\lambda=\lambda_{n}=2 P(0,1)+2 n \pi i$ and that $\mu_{2}\left(x, \lambda_{n}\right)=$ $\mu_{1}\left(x, \lambda_{n}\right) \exp [-P(0,1)]$ for $\lambda_{n}=2 P(0,1)+2 n \pi i$.

Now define

$$
\nu_{1}=\mu_{1} \exp (-\lambda x), \quad \nu_{2}=\mu_{2} \exp (-\lambda x), \quad \varphi_{1}=p \nu_{1}+\nu_{1}^{\prime}, \quad \varphi_{2}=p \nu_{2}+\nu_{2}^{\prime},
$$

$h(x, \lambda)=\frac{\mu_{2}(x)}{K(\lambda)} \int_{0}^{x} f(\xi) \varphi_{1}(\xi) d \xi, \quad j(x, \lambda)=\frac{\mu_{1}(x)}{K(\lambda)} \int_{x}^{1} f(\xi) \varphi_{2}(\xi) d \xi$.

.From (4a) it is also clear that,

$$
\begin{gathered}
\frac{1}{2 \pi i} \oint h(x, \lambda) d \lambda+\frac{1}{2 \pi i} \int j(x, \lambda) d \lambda=\sum_{-\infty}^{\infty} \mu_{1}\left(x, \lambda_{n}\right) \int_{0}^{1} f(\xi)\left[\frac{p(\xi) \nu_{2}\left(\xi, \lambda_{n}\right)+\nu_{2}^{\prime}\left(\xi, \lambda_{n}\right)}{K^{\prime}\left(\lambda_{n}\right)}\right] d \xi \\
=\sum_{-\infty}^{\infty} \mu_{2}\left(x, \lambda_{n}\right) \int_{0}^{1} f(\xi)\left[\frac{p(\xi) \nu_{1}\left(\xi, \lambda_{n}\right)+\nu_{1}^{\prime}\left(\xi, \lambda_{n}\right)}{K^{\prime}\left(\lambda_{n}\right)}\right] d \xi,
\end{gathered}
$$

where now $\lambda_{n}=2 P(0,1)+2 n \pi i$.

From the above definitions we obtain

$$
\begin{gathered}
h(x, \lambda)=\frac{\exp [\lambda(x-1)-P(1, x)]-\exp [P(1, x)]}{\exp [-P(0,1)]-\exp [-\lambda+P(0,1)]} \\
\cdot \int_{0}^{x} f(\xi)\left[\exp [-\lambda \xi+P(0, \xi)]-\frac{2 p \exp [-\lambda \xi-P(0, \xi)]}{\lambda}\right] d \xi, \\
h(x, \lambda)=A(x, \lambda) \int_{0}^{x} f(\xi)\left[\exp [-\lambda \xi+P(0, \xi)]+O\left(\frac{e^{-\lambda \xi}}{\lambda}\right)\right] d \xi, \quad \operatorname{Re} \quad \lambda>0,
\end{gathered}
$$

where

$$
A(x, \lambda)=\frac{\exp [\lambda(x-1)-P(1, x)]-\exp [P(1, x)]}{\exp [-P(0,1)]-\exp [-\lambda+P(0,1)]}
$$

Equation (8) may be written as

$h(x, \lambda)=B(x, \lambda) \int_{0}^{x} f(\xi)\left[\exp [\lambda(x-\xi)+P(0, \xi)]-\frac{2 p \exp [\lambda(x-\xi)+P(0, \xi)]}{\lambda}\right] d \xi,(9)$

where

$$
B(x, \lambda)=\frac{\exp [-P(1, x)]-\exp [(1-x)+P(1, x)]}{\exp [\lambda-P(0,1)]-\exp [P(0,1)]},
$$

and from (9) we have

$h(x, \lambda)=B(x, \lambda) \int_{0}^{x} f(\xi)\left[\exp [\lambda(x-\xi)+P(0, \xi)]+O\left(\frac{\exp [\lambda(x-\xi)]}{\lambda}\right)\right] d \xi . \quad \operatorname{Re} \quad \lambda<0$

Similarly

$$
\begin{aligned}
j(x, \lambda)=\frac{\exp [\lambda x-P(0, x)]-\exp [P(0, x)]}{\exp [-P(0,1)]-\exp [-\lambda+P(0,1)]} \\
\quad \cdot \int_{x}^{1} f(\xi)\left[\exp [-\lambda \xi+P(1, \xi)]-\frac{2 p \exp [-\lambda \xi+P(1, \xi)]}{\lambda}\right] d \xi \\
=C(x, \lambda) \int_{x}^{1} f(\xi)\left[\exp \left[\lambda(x-\xi)+P(1, \xi)-\frac{2 p \exp [\lambda-\xi+P(1, \xi)]}{\lambda}\right] d \xi\right.
\end{aligned}
$$


where

$$
C(x, \lambda)=\frac{\exp [-P(0, x)]-\exp [-\lambda x+P(0, x)]}{\exp [-P(0,1)]-\exp [-\lambda+P(0,1)]}
$$

Consequently

$j(x, \lambda)=C(x, \lambda) \int_{x}^{1} f(\xi)\left[\exp [\lambda(x-\xi)+P(1, \xi)]+O\left(\frac{\exp [\lambda(x-\xi)]}{\lambda}\right)\right] d \xi, \operatorname{Re} \lambda>0$

We may write $j(x, \lambda)$ as

$$
\begin{aligned}
j(x, \lambda)=\frac{\exp [\lambda x-P(0, x)]-\exp [P(0, x)]}{\exp [\lambda-P(0,1)]-\exp [P(0,1)]} \\
\cdot \int_{x}^{1} f(\xi)\left[\exp [\lambda(1-\xi)+P(1, \xi)]-\frac{2 p \exp [\lambda(1-\xi)+P(1, \xi)]}{\lambda}\right] d \xi
\end{aligned}
$$

and obtain

$$
j(x, \lambda)=D(x, \lambda) \int_{x}^{1} f(\xi)\left[\exp \left[\lambda(1-\xi)+P(1, \xi)+O\left(\frac{e^{\lambda(1-\xi)}}{\lambda}\right)\right] d \xi, \quad \text { Re } \quad \lambda<0\right.
$$

where

$$
D(x, \lambda)=\frac{\exp [\lambda x-P(0, x)]-\exp [P(0, x)]}{\exp [\lambda-P(0,1)]-\exp [P(0,1)]}
$$

As previously shown

$$
\begin{aligned}
\int_{0}^{x} f(\xi) 0\left(\frac{e^{-\lambda \xi}}{\lambda}\right) d \xi & =O\left(\frac{e^{-\lambda \xi}}{\lambda}\right), \\
\int_{0}^{x} f(\xi) 0\left(\frac{e^{\lambda(x-\xi)}}{\lambda}\right) d \xi & =O\left(\frac{e^{\lambda(x-\xi)}}{\lambda}\right), \\
\int_{x}^{1} f(\xi) 0\left(\frac{e^{\lambda(1-\xi)}}{\lambda}\right) & =O\left(\frac{e^{\lambda(1-\xi)}}{\lambda}\right),
\end{aligned}
$$

and these integrals tend to zero when integrated over their contours.

Also,

$$
\begin{aligned}
& \lim _{|\lambda| \rightarrow \infty} A(x, \lambda)=-\exp [P(0, x)] \text { for } \operatorname{Re} \lambda>0, \\
& \lim _{|\lambda| \rightarrow \infty} B(x, \lambda)=-\exp [-P(0, x)] \text { for } \operatorname{Re} \lambda<0, \\
& \lim _{|\lambda| \rightarrow \infty} C(x, \lambda)=\exp [P(x, 1)] \text { for } \operatorname{Re} \lambda>0, \\
& \lim _{|\lambda| \rightarrow \infty} D(x, \lambda)=\exp [-P(x, 1)] \text { for } \operatorname{Re} \lambda<0 .
\end{aligned}
$$

From these results we conclude that

$$
\begin{aligned}
\frac{1}{2 \pi i} \oint H(x, \lambda) d \lambda+\frac{1}{2 \pi i} \oint J(x, \lambda) d \lambda= & \frac{1}{2 \pi i} \oint h(x, \lambda) d \lambda \\
& +\frac{1}{2 \pi i} \oint j(x, \lambda) d \lambda \text { for }|\lambda| \rightarrow \infty
\end{aligned}
$$


and consequently

$$
\sum_{-\infty}^{\infty} a_{n} u_{n}(x)=\sum_{-\infty}^{\infty} \mu_{1}\left(x, \lambda_{n}\right) \int_{0}^{1} f(\xi) \frac{\left[p \nu_{2}\left(\xi, \lambda_{n}\right)+\nu_{2}^{\prime}\left(\xi, \lambda_{n}\right)\right]}{K^{\prime}\left(\lambda_{n}\right)} d \xi,
$$

where

$$
K^{\prime}\left(\lambda_{n}\right)=\left[\frac{d}{d \lambda} K(\lambda)\right]_{\lambda_{n}}=\frac{\exp [-P(0,1)]}{\lambda_{n}} .
$$

Using $\lambda_{n}=2 P(0,1)+2 n \pi i$ we obtain

$$
p \nu_{2}\left(\xi \lambda_{n}\right)+\nu_{2}^{\prime}\left(\xi, \lambda_{n}\right)=\exp \left[-\lambda_{n} \xi+P(1, \xi)\right]-\frac{2 p \exp \left[-\lambda_{n} \xi+P(1, \xi)\right]}{\lambda_{n}}
$$

and therefore

$$
\sum_{-\infty}^{\infty} a_{n} u_{n}(x)=\sum_{-\infty}^{\infty}\left[\mu_{1}\left(x, \lambda_{n}\right) \int_{0}^{1} f(\xi) \frac{\exp \left[-\lambda_{n} \xi+P(1, \xi)\right]}{K^{\prime}\left(\lambda_{n}\right)} d \xi-T_{n}\right],
$$

where

$$
T_{n}=\mu_{1}\left(x, \lambda_{n}\right) \int_{0}^{1} 2 p f(\xi) \frac{\exp \left[-\lambda_{n} \xi+P(1, \xi)\right]}{\lambda_{n} K^{\prime}\left(\lambda_{n}\right)} d \xi
$$

also

$$
\frac{\mu_{1}\left(x, \lambda_{n}\right)}{K^{\prime}\left(\lambda_{n}\right)} \int_{0}^{1} f(\xi) \exp \left[-\lambda_{n} \xi+P(1, \xi)\right] d \xi=\beta_{n} \exp g(x)(\exp 2 n \pi i x)-\beta_{n} \exp [P(0, x)],
$$

where

$$
g(x)=2 x \int_{0}^{1} p d t-\int_{0}^{x} p d t
$$

and $\beta_{n}$ is the complex Fourier coefficient

$$
\int_{0}^{1} f(\xi) \exp [-g(\xi)] \exp (-2 n \pi i \xi) d \xi
$$

We conclude then that

$$
\sum_{-\infty}^{\infty} a_{n} u_{n}(x)=\sum_{-\infty}^{\infty}\left[\beta_{n} \exp g(x)[\exp 2 n \pi i x]-\beta_{n} \exp [P(0, x)]-T_{n}\right] .
$$

Now

$$
\begin{aligned}
\sum_{-\infty}^{\infty} T_{n}=\frac{1}{2 \pi i} \oint d \lambda\left[\int_{0}^{x} \frac{2 p f(\xi) \exp [-\lambda \xi+P(0, \xi)]}{\lambda K(\lambda)} \mu_{2}(x, \lambda) d \xi\right. \\
\left.\quad+\int_{x}^{1} \frac{2 p f(\xi) \exp [-\lambda \xi+P(1, \xi)]}{\lambda K(\lambda)} \mu_{1}(x, \lambda) d \xi\right] .
\end{aligned}
$$

From the results of (8) and (9) both of the integrals in (12) tend to zero when the radius of the contour tends to infinity and we conclude that

$$
\sum_{-\infty}^{\infty} T_{n}=0
$$


Now, if $\sum_{-\infty}^{\infty} b_{n} \exp (2 n \pi i x)$ converges for $0<x<1$, then $\sum_{-\infty}^{\infty} b_{n}$ converges also and (11) may be written as

$$
\sum_{-\infty}^{\infty} a_{n} u_{n}(x)=\left[\exp g(x) \sum_{-\infty}^{\infty} \beta_{n} \exp (2 n \pi i x)\right]-\left[\exp [P(0, x)] \sum_{-\infty}^{\infty} \beta_{n}\right],
$$

since $\sum_{-\infty}^{\infty} T_{n}=0$.

Now, if

$$
\sum_{-\infty}^{\infty} \exp (2 n \pi i x) \int_{0}^{1} f(\xi) \exp (-2 n \pi i \xi) d \xi=t(x), \quad 0<x<1
$$

then

$$
\exp g(x) \sum_{-\infty}^{\infty} \beta_{n} \exp (2 n \pi i x)=\exp g(x)[t(x) \exp [-g(x)]]=t(x) .
$$

Now $\sum_{-\infty}^{\infty} \beta_{n}$ converges to some value, say $t_{0}$, and therefore

$$
\sum_{-\infty}^{\infty} a_{n} u_{n}(x)=t(x)-t_{0} \exp [P(0, x)]
$$

If our Fourier series converges uniformly to $t(x)$ for $0<x<1$, clearly then the convergence of (14) is uniform and our theorem is proved.

\section{References}

1. Bernard Friedman and L. I. Mishoe, Eigenfunction expansions associated with a non-self-adjoint differential equation, Pacific Journal of Mathematics, 6, 249-270 (1956)

2. E. W. Hobson, Theory of functions of a real variable and theory of fourier series, Dover Publications, New York, 1957, pp. 771-772

3. E. L. Ince, Ordinary differential equations, Dover Publications, New York, 1956, pp. 276-278 